\title{
Biblioteca Infantil Miriam Álvarez Brenes: perspectiva interdisciplinaria de los talleres impartidos de 2000 al 2009
}

\author{
Infantile Library Miriam Álvarez Brenes: \\ Interdisciplinary Perspective of the Given Workshops \\ from 2000 to 2009
}

\author{
Máster María Marjorie Mora Valverde* \\ Escuela de Bibliotecología, Documentación e Información, \\ Universidad Nacional (UNA)
}

Recibido: 19 de febrero 2016 Aceptado: 16 de agosto 2016

Corregido: 29 de agosto 2016 Publicado: 30 de noviembre 2016

\begin{abstract}
Resumen
En este artículo se presentan los principales resultados de una investigación que analiza los aportes de la biblioteca infantil a la población de las comunidades de Jardines Universitarios 1 y 2 de Heredia y de otras comunidades en la provincia, por medio de los talleres educativos. Estos talleres se han caracterizado por la valiosa participación de estudiantes de diferentes especialidades, como la educación y la psicología, integrados a la labor bibliotecológica en beneficio de la comunidad usuaria.

En el período del año 2000 al 2009 se impartieron en total noventa talleres: 86 dirigidos a la población infantil, y 4 a las familias de la comunidad. También se realizaron 11 charlas, 1 para la población infantil y 10 para las familias. Del total de talleres recibidos por los menores, los de idiomas se han impartido en mayor cantidad y comprenden un 18,6\%, seguidos por los de promoción de la lectura con un $16,3 \%$ y motivos navideños con el mismo porcentaje y apresto escolar con el 12,8\%; estos temas comprenden el 64,4\% de los talleres.
\end{abstract}

Palabras clave: Biblioteca infantil, Interdisciplinariedad, Talleres, Bibliotecología

\section{bibliotecas}




\begin{abstract}
This article presents the main results of a study that analyzes the contributions of the children's library to the people of the communities of University Gardens 1 and 2 of Heredia and other communities in the province, through educational workshops. These workshops have been characterized by the meaningful participation of students from different specialties, such as education and psychology, integrated into the work library science for the benefit of the user community. In the period from 2000 to 2009 were given a total of 90 workshops, 86 to children's and four families in the community. Also, there were 11 talks, one on children and ten families. Workshops of the total received by children, the language has been taught in more and comprise $18,6 \%$, followed by promotion of reading with $16,3 \%$ and Christmas themes with the same percentage and the school readiness $12,8 \%$, these issues comprise $64,4 \%$ of the workshops.
\end{abstract}

Keywords: Children's library, Interdisciplinary, Workshops, Library

\title{
I. Introducción ${ }^{1}$
}

El presente artículo resume los principales resultados del proyecto de investigación titulado Características, factores y beneficios del trabajo interdisciplinario: caso Biblioteca Infantil Miriam Álvarez Brenes, finalizado en el año 2011. Este proyecto se originó de la necesidad de investigar las particularidades del trabajo interdisciplinario que se realiza en la biblioteca, desde la perspectiva de los profesionales que han participado en los talleres dirigidos a la población infantil con la finalidad de fortalecer, beneficiar y solventar los factores que limitan la interdisciplinariedad. Los objetivos de la investigación son los siguientes:

- Realizar un estudio del trabajo interdisciplinario en la Biblioteca Infantil Miriam Álvarez.

- Caracterizar a los instructores que han realizado trabajos interdisciplinarios en la Biblioteca Infantil.

- Describir los talleres que se han realizado en la Biblioteca Infantil. 
- Conocer los logros que causan las actividades interdisciplinarias en la población infantil que asiste a la Biblioteca Infantil.

- Determinar los factores que limitan y benefician el trabajo interdisciplinario en la Biblioteca Infantil.

- Conocer otras áreas profesionales para ampliar el trabajo interdisciplinario existente.

Esta investigación se concentró en los talleres educativos realizados regularmente en la biblioteca, los que se caracterizan por la participación de estudiantes universitarios de diferentes especialidades que, con nombramiento de horas asistente, se integraron a la labor bibliotecológica en beneficio de la comunidad usuaria. Estos talleres, junto con los préstamos en sala y a domicilio, son las principales actividades formativas de la biblioteca; no obstante, los talleres son los únicos susceptibles a ser analizados desde la perspectiva interdisciplinaria por el aporte de los estudiantes de diferentes especialidades.

En este estudio es de especial interés analizar las características de los talleres educativos y del personal instructor y destacar los aportes que, desde una perspectiva interdisciplinaria, se han producido en la comunidad de Jardines Universitarios 1 y 2 de Heredia. Para estudiar el proceso interdisciplinario de la biblioteca, se hace énfasis en la percepción del personal instructor desde su especialidad, condición relevante para la aproximación del objeto de estudio ${ }^{2}$.

\section{a. La Biblioteca Infantil Miriam Álvarez Brenes}

La Biblioteca Infantil Miriam Álvarez Brenes (BIMIAL) es un proyecto de extensión social de la Escuela de Bibliotecología, Documentación e Información (EBDI) de la Universidad Nacional (UNA), que nació en el año 1994 para contribuir con el mejoramiento de las condiciones sociales de los habitantes de dos comunidades marginales: Jardines Universitarios 1 y 2, que en la década de 1990 se ubicaban en los alrededores de la Universidad Nacional. Este proyecto representa los

\section{bibliotecas}


valores humanistas que motivaron la creación de la universidad y es un medio para proyectar el quehacer de la institución a la comunidad, de acuerdo con lo establecido en el capítulo segundo del Estatuto Orgánico de la UNA.

El 26 de setiembre de 1996 se inauguró la planta física que albergaría esta biblioteca, cumpliéndose así un objetivo que requirió tres años de trabajo (1994 a 1996). En el mes de setiembre de 2011 la biblioteca infantil cumplió 15 años de servir a la comunidad, tiempo durante el cual ha realizado diferentes actividades educativas para atraer a los infantes y a los padres y las madres de familia.

La biblioteca ha atendido en sus instalaciones las consultas del material bibliográfico de la colección infantil, las delegaciones infantiles de kínder y escuelas de la provincia y ha ofrecido gran cantidad de talleres sobre diferentes temáticas a la numerosa población infantil que la ha visitado y disfrutado de un "viaje de ensueño en su vagón mágico". En el marco de este proyecto se ha integrado en las actividades de la biblioteca a las familias de la comunidad y se han ofrecido charlas a los padres y a las madres sobre temas que benefician su crecimiento personal y familiar.

\section{b. El trabajo interdisciplinario de la bibliotecología}

La bibliotecología se enriquece con los aportes que recibe de disciplinas como educación, psicología, informática, estadística, entre otras. Para Rodríguez (2006): "la bibliotecología necesita de los conocimientos de otras disciplinas para avanzar, al igual que otras áreas del conocimiento requieren de los saberes de la bibliotecología” (p.13). En la actualidad es necesaria la interacción entre las diversas ciencias para "encontrar explicaciones más profundas, más rigurosas y mejor fundamentadas que permitan ampliar la comprensión de los fenómenos investigados y hacer avanzar a la bibliotecología y a las demás ciencias" (Rodríguez, 2006, p.14).

Las disciplinas pueden y deben complementarse, tal situación significa que un proceso se analiza y se explica desde diferentes perspectivas disciplinarias. A partir del trabajo conjunto entre especialistas de diversas disciplinas, 
en el cual cada quien conoce en profundidad el campo de su especialidad, se producen aportaciones de gran valor que contribuyen a generar y a consolidar el nuevo conocimiento, que es lo que en verdad se llama interdisciplinariedad. La interdisciplinariedad se presenta cuando se producen interacciones que relacionan diversas disciplinas entre sí (Gorbea, 2006).

En un grupo de trabajo en el cual cada integrante es experto en su propia disciplina hay multidisciplinariedad. La interdisciplinariedad existe cuando se produce la integración de los diferentes enfoques disciplinarios para el abordaje de un problema específico. En la investigación multidisciplinaria solo se suman los aportes de cada especialista desde su disciplina particular. Es en la delimitación de la problemática de investigación en la que se produce la integración disciplinar: la interdisciplinariedad se presenta como un procedimiento de trabajo que propone integrar o coordinar conceptos, métodos y conclusiones de dos o más disciplinas con el objeto de llegar a un resultado (García, 2006).

\section{Materiales y métodos}

Esta investigación es de tipo exploratorio y se enmarca dentro del enfoque de investigación mixto. La caracterización de los instructores y la descripción de los talleres se realizaron por medio de técnicas de análisis cuantitativo, mientras que el estudio de la percepción de los instructores sobre el trabajo interdisciplinario de la biblioteca requirió el uso de técnicas de recolección y de análisis cualitativas.

En esta investigación se utilizaron las siguientes fuentes de información:

- Los informes anuales de labores de las autoridades de la biblioteca recibidos en la Dirección de la EBDI, de 1996 hasta el año 2009. El carácter oficial de los informes anuales permitió considerarlos como documentos idóneos para obtener la información de los talleres y de las charlas, los temas abordados y los instructores de los talleres y las charlas, a quienes interesaba investigar.

- La entrevista realizada al grupo de instructores seleccionado.

\section{bibliotecas}


De los informes anuales de la biblioteca se obtuvieron los datos que se presentan en el apartado de resultados, los cuales se clasificaron según la población meta que recibió el taller o la charla: población infantil y familias de la comunidad. Para caracterizar a los instructores se elaboraron dos cuadros con este personal clasificado según el total de talleres y charlas impartidas, la especialidad de los instructores y el tipo de trabajo o relación que tenían con la biblioteca.

La otra fuente de información consistió en una encuesta que se realizó al personal instructor de los talleres que fue identificado en el estudio documental. En esta investigación se utilizó la entrevista semi-estructurada como técnica de recolección de la información, pero se adaptó la técnica a las características del personal instructor, el cual posee un nivel de escolaridad universitaria y por tanto puede escribir sus propias respuestas. Para orientar la entrevista se utilizó un cuestionario (anexo 4 del informe final, ver Nota ${ }^{3}$ del presente artículo), dividido en tres secciones: una sección con preguntas cerradas sobre las características personales de los instructores y la segunda y tercera sección sobre los talleres, las actividades realizadas y la integración de la bibliotecología con las diferentes disciplinas del persona instructor.

De noventa talleres realizados, setenta y cinco fueron impartidos por el personal de planta de la biblioteca y por los estudiantes contratados por horas asistente; con base en esta información, se seleccionó el personal instructor que cumplía con los siguientes requisitos:

- Personal de planta de la biblioteca y estudiantes horas asistente que se desempeñaron como instructores.

- Estudiantes asistentes que tuvieron una permanencia en el proyecto de por lo menos un año.

De esta forma, se seleccionó a 13 personas para entrevistar, de las cuales no se localizó a tres, por esta razón los resultados se presentan para 10 personas. Por el amplio periodo del estudio, era de esperar que hubiera dificultades para localizar a los instructores más antiguos. 


\section{Resultados}

En el periodo del año 2000 al 2009 se impartió un total de noventa talleres, ochenta y seis para la población infantil y cuatro a las familias de la comunidad de Jardines 1 y 2 de Heredia. También, se realizaron 10 charlas, 1 para la población infantil y 9 a las familias.

a. Talleres y charlas impartidos a la población infantil de la comunidad de Jardines 1 y 2 de Heredia del año 2000 al 2009.

En este período se impartió a la población infantil de la comunidad un total de ochenta y seis talleres (Cuadro 1) y una charla. A los talleres asistieron 910 niños y niñas con edades entre 3 y 12 años (Cuadro 4). La charla El Mundo de la Red Internet se impartió en el año 2006 por la instructora Fabiola Campos y contó con la participación de 12 niños y niñas de 8 a 12 años.

\section{Cuadro 1}

Biblioteca Infantil: total de talleres impartidos y población infantil asistente de la comunidad de Jardines 1 y 2 de Heredia, según año del taller 2000 al 2009

\begin{tabular}{ccc}
\hline Año & Total de talleres & Total de la población asistente \\
\hline 2000 & 6 & 91 \\
\hline $2002 \frac{1}{}$ & 10 & 107 \\
\hline 2003 & 10 & 135 \\
\hline 2004 & 7 & 77 \\
\hline 2005 & 15 & 132 \\
\hline 2006 & 10 & 97 \\
\hline 2007 & 10 & 96 \\
\hline 2008 & 7 & 80 \\
\hline 2009 & 11 & 95 \\
\hline Total & $\mathbf{8 6}$ & $\mathbf{9 1 0}$ \\
\hline
\end{tabular}

1 El informe del año 2001 no hace referencia a la realización de talleres.

Fuente: elaboración propia a partir de los datos de los informes anuales de la Biblioteca Infantil Miriam Álvarez Brenes.

\section{bibliotecas}


El Cuadro 2 muestra que la mayor cantidad de talleres corresponden a temas relacionados con los idiomas, en primer lugar, seguido por la promoción de la lectura y los motivos navideños, en segundo lugar, y los talleres sobre el apresto escolar, en tercer lugar. Estos cuatro temas comprenden el 64\% del total de talleres impartidos en el periodo de 2000 al 2009, temas que concentran la mayor participación de la población infantil, un 64\%.

Durante el periodo en estudio, los talleres sobre idiomas ocupan el primer lugar en la cantidad de talleres impartidos en la biblioteca. Del total de talleres realizados el $18,6 \%$ corresponde a idiomas y cuentan con una participación del $14,4 \%$ del total de la población. De los 16 talleres de idiomas, 13 fueron en inglés, 2 en francés y 1 en mandarín. Los instructores fueron estudiantes de la enseñanza del inglés, de francés y de enseñanza de la filosofía, respectivamente.

La importancia de aprender un segundo o tercer idioma y el incremento de la demanda de los cursos de idiomas en los últimos años, motivó que se impartieran estos talleres en la biblioteca. Estudiar un nuevo idioma abre muchas posibilidades de aprendizaje y proporciona una mayor variedad de conocimientos, además, si desde temprana edad la población infantil empieza a conocer y a hablar otro idioma, este le resultará más fácil de aprender.

El segundo tema con mayor cantidad de talleres es la promoción de la lectura con un 16,3\%; a estos talleres asistió el 14,1\% del total de asistentes. Estos talleres fueron impartidos por instructoras con especialidad en bibliotecología y con menor participación de educación preescolar y enseñanza de la filosofía. Los talleres sobre motivos navideños ocupan, también, un segundo lugar, comprenden el 16,3\% del total de talleres realizados en la biblioteca y en ellos han participado el 15,7\% de la población infantil asistente. Los talleres tienen una participación similar de instructoras de la disciplina de la bibliotecología y de enseñanza preescolar.

El 12,8\% de los talleres impartidos tuvo la finalidad de contribuir con el apresto escolar de los infantes y representó una asistencia del 12,4\% del total de asistentes a los talleres. La mayoría de las instructoras de 


\section{Cuadro 2}

Biblioteca infantil: total de talleres impartidos y población infantil asistente de la comunidad de Jardines 1 y 2 de Heredia, según tema del taller 2000 al 2009

\begin{tabular}{ccccc}
\hline & \multicolumn{2}{c}{ Total de talleres } & \multicolumn{2}{c}{$\begin{array}{c}\text { Total de población infantil } \\
\text { asistente }\end{array}$} \\
\cline { 2 - 5 } Tema de los talleres & $\begin{array}{c}\text { Número de } \\
\text { talleres }\end{array}$ & $\begin{array}{c}\text { Porcentaje } \\
\text { de talleres }\end{array}$ & $\begin{array}{c}\text { Número de } \\
\text { población }\end{array}$ & $\begin{array}{c}\text { Porcentaje de } \\
\text { población }\end{array}$ \\
\hline Total & $\mathbf{8 6}$ & $\mathbf{1 0 0 , 0}$ & $\mathbf{9 1 0}$ & $\mathbf{1 0 0 , 0}$ \\
\hline Idiomas & 16 & 18,6 & 131 & 14,4 \\
\hline $\begin{array}{c}\text { Promoción de la } \\
\text { lectura }\end{array}$ & 14 & 16,3 & 128 & 14,1 \\
\hline Motivos navideños & 14 & 16,3 & 143 & 15,7 \\
\hline Apresto escolar & 11 & 12,8 & 113 & 12,4 \\
\hline Manualidades & 6 & 7,0 & 48 & 5,3 \\
\hline Desarrollo personal & 5 & 5,8 & 43 & 4,7 \\
\hline Técnicas de estudio & 5 & 5,8 & 35 & 3,8 \\
\hline Juegos & 4 & 4,7 & 39 & 4,3 \\
\hline $\begin{array}{c}\text { Deberes, derechos y } \\
\text { valores }\end{array}$ & 4 & 4,7 & 31 & 3,4 \\
\hline $\begin{array}{c}\text { Aprendizaje de la } \\
\text { ciencia y la tecnología }\end{array}$ & 3 & 3,5 & 27 & 3,0 \\
\hline Ecología & 3 & 3,5 & 23 & 2,5 \\
\hline $\begin{array}{c}\text { Prevención de Acci- } \\
\text { dentes }\end{array}$ & 1 & 1,0 & 14 & 1,5 \\
\hline No se indica el tema 1/ & 0 & 0,0 & & 135 \\
\hline
\end{tabular}

1/ En un taller no se indica la especialidad de la instructora.

Fuente: elaboración propia a partir de los datos de los informes anuales de la Biblioteca Infantil Miriam Álvarez Brenes.

estos talleres tienen la especialidad en bibliotecología, solo una es de preescolar. En el ámbito educativo el apresto escolar es considerado de

\section{bibliotecas}


gran importancia para que la población infantil alcance determinadas destrezas que le permitan adaptarse satisfactoriamente al sistema educativo formal del kínder y la enseñanza primaria.

El resto de los temas de los talleres, un 36\% estuvo referido a los temas de manualidades (7\%) para contribuir con el desarrollo de destrezas, principalmente motoras; el desarrollo personal (5,8\%); hábitos de estudio $(5,8 \%)$ para que la población infantil que ya ingresó a primaria mejore su rendimiento; juegos $(4,7 \%)$ para el desarrollo físico y emocional; conocimiento de los deberes y los derechos (4,7\%); aprendizaje de la ciencia y la tecnología (3,5\%); el desarrollo de una conciencia ecológica $(3,5 \%)$ y la prevención de accidentes $(1,0 \%)$.

En el Cuadro 3 se observa que del total de talleres, el 34,9\% fue impartido por instructoras con especialidad en bibliotecología, un 23,3\%, en enseñanza preescolar $y$, en tercer lugar, un $15,1 \%$ de instructoras de enseñanza del inglés. En el Cuadro 4 se observa que hay mayor cantidad de talleres dirigidos a los menores de 3 a 6 años. La participación en los talleres es mayor en la población infantil de menor edad y en promedio participan 11 infantes por taller, mientras que en los de mayor edad el promedio es de aproximadamente 9.

\section{Cuadro 3}

Biblioteca infantil: total de talleres impartidos y población infantil asistente de la comunidad de Jardines 1 y 2 de Heredia, según especialidad de los instructores, 2000 al 2009

\begin{tabular}{ccccc}
\hline \multirow{2}{*}{$\begin{array}{c}\text { Especialidad } \\
\text { de la persona instructora }\end{array}$} & \multicolumn{2}{c}{ Total de talleres } & \multicolumn{2}{c}{$\begin{array}{c}\text { Total de población infantil } \\
\text { asistente }\end{array}$} \\
\cline { 2 - 5 } & $\begin{array}{c}\text { Número de } \\
\text { talleres }\end{array}$ & $\begin{array}{c}\text { Porcentaje de } \\
\text { talleres }\end{array}$ & $\begin{array}{c}\text { Número de la } \\
\text { población }\end{array}$ & $\begin{array}{c}\text { Porcentaje de } \\
\text { la población }\end{array}$ \\
\hline Total & $\mathbf{8 6}$ & $\mathbf{1 0 0 , 0}$ & $\mathbf{9 1 0}$ & $\mathbf{1 0 0 , 0}$ \\
\hline Bibliotecología & 30 & 34,9 & 340 & 37,4 \\
\hline Preescolar & 20 & 23,3 & 195 & 21,4 \\
\hline Enseñanza del inglés & 13 & 15,1 & 98 & 10,8 \\
\hline Psicología & 5 & 5,8 & 54 & 5,9 \\
\hline Enseñanza de la filosofía & 5 & 5,8 & 44 & 4,8 \\
\hline
\end{tabular}




\begin{tabular}{ccccc}
\hline Enseñanza del francés & 2 & 2,3 & 24 & 2,6 \\
\hline Artes plásticas & 1 & 1,2 & 10 & 1,1 \\
\hline $\begin{array}{c}\text { Sin especialidad } \\
\text { conocida }\end{array}$ & 10 & 11,6 & 145 & 15,9 \\
\hline
\end{tabular}

Fuente: elaboración propia a partir de los datos de los informes anuales de la Biblioteca Infantil Miriam Álvarez Brenes

\section{Cuadro 4}

Biblioteca infantil: total de talleres impartidos y población infantil asistente de la comunidad de Jardines 1 y 2 de Heredia, por grupos de edad, según año del taller 2002 y 2004 al 2009 (solo incluye los casos con edad conocida)

\begin{tabular}{ccccccc}
\hline \multirow{2}{*}{ Año } & \multicolumn{3}{c}{ Número de talleres } & \multicolumn{3}{c}{ Total de población infantil asistente } \\
\cline { 2 - 7 } & Total & 3 a 6 años & 6 a 12 años & Total & 3 a 6 años & 6 a 12 años \\
\hline 2002 & 10 & 4 & 6 & 107 & 49 & 58 \\
\hline 2004 & 7 & 6 & 1 & 77 & 69 & 8 \\
\hline 2005 & 15 & 6 & 9 & 132 & 56 & 76 \\
\hline 2006 & 10 & 5 & 5 & 97 & 49 & 48 \\
\hline 2007 & 10 & 6 & 4 & 96 & 67 & 29 \\
\hline 2008 & 7 & 4 & 3 & 80 & 48 & 32 \\
\hline 2009 & 11 & 8 & 3 & 95 & 75 & 20 \\
\hline Total ${ }^{1 /}$ & $\mathbf{7 0}$ & $\mathbf{3 7}$ & $\mathbf{3 1}$ & $\mathbf{6 8 4}$ & $\mathbf{4 1 3}$ & $\mathbf{2 7 1}$ \\
\hline
\end{tabular}

${ }^{1 /}$ No incluye 16 talleres con 226 niños y niñas porque no se indica la edad.

Fuente: elaboración propia a partir de los datos de los informes anuales de la Biblioteca Infantil Miriam Álvarez Brenes.

\section{b. Talleres y charlas impartidos a los padres y madres de familia de la comunidad de Jardines 1 y 2 de Heredia del 2001 al 2009}

Como parte de la integración de la familia en las actividades de la población infantil en la biblioteca se realizaron capacitaciones para los padres y las madres de la comunidad de Jardines 1 y 2 de Heredia. Du- 
rante el período del 2001 al 2009 se impartieron 9 charlas y 4 talleres cuya información se presenta en los Cuadros 5 y 6 siguientes.

\section{Cuadro 5}

Biblioteca infantil: total de talleres y charlas impartidos a las familias de la comunidad de Jardines 1 y 2 de Heredia, según año 2001 al 2009

\begin{tabular}{ccc}
\hline Año & Total de talleres & Total de charlas \\
\hline 2001 & 0 & 2 \\
\hline 2004 & 0 & 1 \\
\hline 2005 & 2 & 0 \\
\hline 2006 & 0 & 2 \\
\hline 2007 & 1 & 0 \\
\hline 2008 & 0 & 4 \\
\hline 2009 & 1 & 0 \\
\hline Total & 4 & 9 \\
\hline
\end{tabular}

Fuente: elaboración propia a partir de los datos de los informes anuales de la Biblioteca Infantil Miriam Álvarez Brenes.

\section{Cuadro 6}

Biblioteca infantil: número de talleres y número de charlas impartidos a las familias de la comunidad de Jardines 1 y 2 de Heredia, según especialidad de la instructora, del 2001 al 2009

\begin{tabular}{ccc}
\hline Especialidad & Número de talleres & Número de charlas \\
\hline Bibliotecología & 0 & 5 \\
\hline Psicología & 3 & 4 \\
\hline Orientación (CIDE) & 1 & 0 \\
\hline Total & $\mathbf{4}$ & $\mathbf{9}$ \\
\hline
\end{tabular}

Fuente: elaboración propia a partir de los datos de los informes anuales de la Biblioteca Infantil Miriam Álvarez Brenes. 
En detalle, en la biblioteca se realizaron las siguientes actividades de capacitación para las familias:

- En el año 2001 la licenciada en bibliotecología, Sandra Alpízar Moya, coordinadora del proyecto en esa época, impartió dos charlas:

- La importancia de la Biblioteca Infantil en el desarrollo integral de los niños.

- Los beneficios que ofrece la lectura en los niños.

- En el año 2004 Angie Bolaños y Natalia Rivera, bachilleres en psicología, facilitaron la charla Aprendiendo a manejar el estrés.

- En el año 2005 se impartieron dos talleres:

- Estrategias para la formación de hábitos de lectura en los niños y las niñas. Taller impartido por Laura Trujillo, estudiante de danza y psicología, bajo la supervisión de la aáster Flor Vargas, encargada de la biblioteca.

- Construyamos alternativas para la no violencia en nuestros hogares. Taller impartido a 15 de madres de familia por las estudiantes Ileana Vargas, Jacqueline Vega y Roxana Venegas, como parte de la práctica supervisada de la carrera de psicología, con la orientación de la profesora Mónica Flochova L.

- En el año 2006 se ofrecieron dos charlas a padres y madres de familia:

- ¿Cómo estimular el hábito lector en los niños(as)? impartido por la máster en educación Flor Vargas.

- La biblioteca infantil centro de información y apoyo para los padres de familia con niños en edad escolar, impartido por las estudiantes de psicología Ileana Vargas, Karla Salmerón y Ana Carolina Hernández.

- En el año 2007 se desarrolló un taller sobre Negociación de límites para ser mejores madres y cuidadoras, al que asistieron 16 madres

\section{bibliotecas}


entre 20 y 40 años, impartido por Carolina Hernández Marlene Borbón y Wendy Víquez, estudiantes de psicología.

- En el año 2008 se impartieron cuatro charlas como parte de un curso optativo de estudiantes de psicología y bibliotecología:

- Promoviendo conductas alternativas hacia la no violencia.

- Aspectos principales del desarrollo integral del niño de 3 a 5 años.

- La biblioteca infantil y el vínculo con los padres y madres de familia en la tarea de promoción de lectura en niños.

- Ayudas para seleccionar libros y material especial para niños en edad preescolar y escolar.

- En el año 2009 se impartió un taller titulado Fortaleciendo a la familia, con una duración de cinco sesiones, a cargo de las instructoras Ligia Ramírez e Ivannia Núñez de la carrera de orientación del CIDE, coordinado por la máster en educación Flor Vargas.

c. Personas instructoras y colaboras de los talleres y charlas impartidos en la biblioteca infantil

La biblioteca inició labores con la señora Sandra Alpízar, licenciada en bibliotecología y coordinadora del proyecto; la señora Flor Vargas Bolaños, bachiller en bibliotecología y encargada de la biblioteca, y la estudiante de bibliotecología contratada como horas asistente Jorleny Valerio. Además de estas funcionarias, se contó con la colaboración de estudiantes universitarios y de los colegios, y de personas de la comunidad quienes colaboraron voluntariamente en diversas funciones. En el Cuadro 7 se presenta la cantidad de talleres y charlas impartidos en la biblioteca infantil, en el período 2000 al año 2009 y el número de personas instructoras según especialidad. 


\section{Cuadro 7}

Biblioteca infantil: personas instructoras, total de talleres y de charlas impartidos a la comunidad de Jardines 1 y 2 de Heredia, según especialidad, del 2000 al 2009

\begin{tabular}{ccccc}
\hline \multirow{2}{*}{ Especialidad } & Total & $\begin{array}{c}\text { Tersonas } \\
\text { instructoras de } \\
\text { los talleres }\end{array}$ & Total & $\begin{array}{c}\text { Personas } \\
\text { instructoras de las } \\
\text { charlas }\end{array}$ \\
\cline { 2 - 5 } & 31 & $7^{\text {a/ }}$ & 6 & 5 \\
\hline Bibliotecología & 20 & 4 & 0 & 0 \\
\hline Preescolar & 13 & 4 & 0 & 0 \\
\hline Enseñanza del inglés I y II Ciclo & 7 & $8^{\text {b/ }}$ & 4 & 7 \\
\hline Psicología & 5 & 1 & 0 & 0 \\
\hline Enseñanza de filosofía & 2 & 1 & 0 & 0 \\
\hline Enseñanza del francés & 1 & 2 & 0 & 0 \\
\hline Orientación (CIDE) & 1 & 1 & 0 & 0 \\
\hline Profesora artes plásticas & 10 & $\ldots$ & 0 & 0 \\
\hline No se indica especialidad & 90 & 28 & 10 & 12 \\
\hline Total & & & & 0 \\
\hline
\end{tabular}

...: no disponible. ${ }^{\mathrm{a} /}$ Incluye tres estudiantes de horas colaboración. ${ }^{\mathrm{b}}$ /Incluye dos estudiantes de práctica supervisada y 2 de horas colaboración.

Fuente: elaboración propia a partir de los datos de los informes anuales de la Biblioteca Infantil Miriam Álvarez Brenes.

De estas personas se destaca que la mayoría pertenecen a carreras del área de la educación (educación preescolar, enseñanza del inglés, enseñanza del francés, enseñanza de la filosofía, orientación y artes plásticas), seguidas por las de bibliotecología y psicología.

En el Cuadro 8 se observan los diferentes tipos de trabajo realizados por el personal de la biblioteca infantil, la mayoría son estudiantes contratados por horas asistente, le siguen estudiantes que realizan horas colaboración en la biblioteca (Ver el anexo 1 del informe completo), en menor cantidad

\section{bibliotecas}


se encuentra la participación de estudiantes que realizan la práctica supervisada, el trabajo comunal y quienes aportan trabajo ad honorem. En el año 2010 la biblioteca tuvo como personal de planta a la encargada Flor Vargas Bolaños, máster en educación y bachiller en bibliotecología, y a la estudiante Marianela Campos, bachiller en bibliotecología.

Las personas instructoras de los talleres y de las charlas son, en su mayoría personal, de planta de la biblioteca y estudiantes nombrados por horas asistente de diversas carreras de la Universidad Nacional. En el Cuadro 8 se muestra a 20 personas que corresponden a esta condición, las cuales han impartido 76 talleres. Este personal corresponde a 18 mujeres y 2 hombres, y la mayoría posee edades que oscilan entre los 20 y 23 al momento de impartir los talleres. Las personas instructoras con otra relación laboral con la biblioteca (horas colaboración, ad honoren, práctica supervisada, trabajo comunal y curso optativo) impartieron en conjunto diez talleres.

Cuadro 8

Biblioteca infantil: personas instructoras y total de talleres y de charlas impartidos a la comunidad de Jardines 1 y 2 de Heredia, según tipo de trabajo, del 2000 al 2009

\begin{tabular}{ccc}
\hline Tipo de trabajo & $\begin{array}{c}\text { Número de personas instructoras } \\
\text { de talleres y charlas }\end{array}$ & Número de talleres ${ }^{1 /}$ \\
\hline Coordinadora del Proyecto & 1 & $0(2)$ \\
\hline Encargada & 1 & $24(1)$ \\
\hline Asistente & 2 & $4(1)$ \\
\hline Horas asistentes & 17 & $48(2)^{\mathrm{a} /}$ \\
\hline Horas colaboración & 6 & $7(0)^{\text {a/ }}$ \\
\hline Ad honorem & 1 & $1(0)$ \\
\hline Práctica supervisada & 3 & $1(0)$ \\
\hline Trabajo comunal & 2 & $1(0)$ \\
\hline Curso optativo & 3 & $0(4)$ \\
\hline Total & 36 & $86(10)$ \\
\hline
\end{tabular}

${ }^{1 / L o s ~ v a l o r e s ~ e n t r e ~ p a r e ́ n t e s i s ~ c o r r e s p o n d e n ~ a l ~ n u ́ m e r o ~ d e ~ c h a r l a s ~ i m p a r t i d a s . ~}{ }^{a /}$ Seis talleres se contabilizan en ambas categorías porque se imparten en conjunto con estudiantes horas asistentes y horas colaboración, para contabilizar el total de talleres efectivamente realizados es necesario restar al total de talleres estos seis.

Fuente: elaboración propia a partir de los datos de los informes anuales de la Biblioteca Infantil Miriam Álvarez Brenes. 
Debido a la mayor participación del personal de planta de la biblioteca y de los estudiantes contratados por horas asistente en la realización de los talleres, estas personas instructoras fueron seleccionadas para las entrevistas, cuyos resultados se describen en la sección siguiente. Como se mencionó en la sección de procedimientos metodológicos, solo fue posible localizar a diez de estas personas seleccionadas para las entrevistas.

De las diez personas entrevistadas de los talleres impartidos en la Biblioteca Infantil Miriam Álvarez Brenes, 9 son mujeres y 1 es hombre; 2 son del área de la bibliotecología, 2 de educación preescolar, 2 de psicología, 2 de orientación, 1 de la enseñanza del inglés y 1 de filosofía. De estas personas, 9 trabajaron como estudiantes asistentes en la biblioteca y 1 es la encargada de la biblioteca, cargo que ocupa desde su creación. Las personas instructoras que fueron estudiantes asistentes, a pesar de ser muy jóvenes, ya terminaron o están terminando sus estudios universitarios y trabajan como docentes en centros educativos: 6 tienen bachillerato y 4 , licenciatura.

d. Percepción de las personas instructoras sobre la labor interdisciplinaria realizada en la biblioteca y el efecto del trabajo en la disciplina de la bibliotecología y en la comunidad de Jardines Universitarios 1 y 2 de Heredia

1. Aportes de las actividades interdisciplinarias en la población infantil que asiste a la Biblioteca Infantil

Los logros de los infantes dependen de su edad y del tipo de taller. En la biblioteca se imparten talleres para la población infantil de 3 a 51/2 o 6 años, que por su corta edad apenas inician el proceso de socialización. A esta población la biblioteca le ofrece la oportunidad de interaccionar con otros infantes de su misma edad. En este espacio aprenden normas para la convivencia en sociedad (dar oportunidad para que hable, prestar un juguete, compartir la merienda, entre otros), algunas nociones sobre matemáticas (grande-pequeño, cerca-lejos, mucho-poco) y el gusto por la lectura.

Desde un proceso lúdico se introduce a la población infantil en la lectoescritura, esto es que se estimula el proceso de lectura de la mano

\section{bibliotecas}


con la escritura. Con respecto a la motivación por la lectura una de las instructoras refiere que: "al final de las sesiones muchos se quedaban y llevaban libros, durante las sesiones se observaba una gran motivación, incluso niños y niñas que las madres hacían referencia que eran muy inquietos trabajan de forma muy adecuada en cada sesión.” El desarrollo del interés por la lectura, por medio del cuento, permite a los infantes interiorizar los contenidos que se desea promover.

En los talleres también se procura que la población infantil adquiera cierto grado de independencia, para que se vaya desapegando de sus padres y madres: "Si en primera instancia los pequeños iban acompañados de sus padres posteriormente son los niños de 10 a 12 años que asisten a los talleres y escogen formar parte." Como señalan las personas instructoras, la biblioteca brinda un espacio para la evolución social de esta población, pues se nota un gran avance al comparar el estado inicial de los infantes con su estado al finalizar el taller.

Las actividades grupales contribuyen a fomentar el trabajo en equipo entre la población infantil. Los de más edad, además de estas normas, tienen la oportunidad de aprender conocimientos más específicos cuando se les imparte algún taller de inglés o el uso de las nuevas tecnologías (en el 2008 se inician talleres sobre alfabetización informacional para la población de 8 a 12 años).

Una de las personas instructoras que trabajó el tema de las relaciones interpersonales reconoce que "al finalizar los infantes lograban interactuar más asertivamente con los demás niños y niñas” y destaca como uno de los logros más significativos "el reconocimiento de sentimientos los cuales se desarrollaron con una técnica lúdica, donde se logró detectar situaciones vivenciadas en los sistemas familiares".

El rescate de la cultura autóctona es de gran importancia en la actualidad, por la influencia de los medios de comunicación en la transmisión de valores, juegos y costumbres de otras culturas; en la biblioteca, por medio de los talleres se trata que la población infantil conozca y practique los juegos de sus padres y madres y de sus antepasados. Sobre este tema una de las personas instructoras escribió: 
Trabajamos un taller con los niños y niñas sobre la recreación dirigida a juegos más tradicionales y menos actuales, para que logren vincularse a sus padres y madres y viceversa, y fomentar en ellos valores que pueden ser aprehendidos y aprendidos en forma constructiva, por ende se trabajó de tal manera con los objetivos de la Orientación, como lo es el desarrollo vocacional en la construcción del proyecto de vida de los niños y niñas. Dentro de los aprendizajes de los niños y niñas pueden destacarse, valores impartidos, destrezas y habilidades, trabajo en equipo, expresión de sentimientos y emociones. Además se trabaja con los padres y madres en Escuela para la Familia, y se potencia la triada Institución-Familia-Niño o Niña.

Las temáticas sobre el medio ambiente contribuyen con la sensibilización por la naturaleza, sobre este tema una persona instructora señaló que la población infantil "se preocupaban por no dejar basura cuando se hacían manualidades". En los últimos años ha adquirido mucha importancia el dominio de un segundo idioma y se ha promovido en todas las escuelas la enseñanza del inglés. En la biblioteca se han impartido talleres para que la población se inicie en la pronunciación y el vocabulario de otro idioma.

La población infantil desde temprana edad aprende a conocer la biblioteca, el uso que se le debe dar y los recursos de que dispone. Y al mismo tiempo ellos desarrollan el interés por investigar, aprovechando los materiales de la biblioteca que determinan sus propios gustos.

2. Elementos de integración social que fomenta la biblioteca

La promoción de la lectura en los infantes siembra la semilla para querer aprender desde la biblioteca o como lo expresa una de las personas instructoras: "En general, y como expresé anteriormente, la evolución social forma parte de los logros más importantes que se dan con la ejecución de los talleres. Entre algunos de los elementos más importantes destacan: a) fomento de una escala de valores (creo que este elemento resume todo), b) primeros contactos con la ética y moral, c) relaciones

\section{bibliotecas}


directas con otros niños y niñas que se encuentran en etapas similares del desarrollo." Además de la contribución al proceso de socialización de los infantes, se han impartido talleres sobre las nuevas tecnologías para procurar cerrar la brecha tecnológica y de comunicación.

Para las personas instructoras en los talleres este proceso de integración social se produce por medio del fomento de:

- Habilidades y valores para la vida, como la tolerancia, el respeto, la seguridad de sí mismo, la autoestima y la autoimagen.

- Actividades para que la población infantil que presentaba un grado significativo de egocentrismo, lograra trabajar con base a ellas y que al final socializara de manera efectiva con los demás compañeros.

- El trabajo desde la integralidad (población infantil, institución, familia) ya que de esta manera se atienden las necesidades en todos los ámbitos en los cuales se desenvuelven los infantes.

- El compartir, el respeto y el ayudar a los demás fueron algunas de las necesidades que se detectaron y, por ende, se trabajó para generar cambios muy significativos en el accionar.

- Gusto por la lectura, pero una lectura significativa, de manera tal que el trabajo que ese día se iba a realizar estuviera acorde con el cuento inicial y se reforzaba constantemente el tema para que lo interiorizaran de la mejor manera.

- Participación directa e indirecta de las familias (fuerza del vínculo familiar).

- Concientización de temas como la diversidad, el medio ambiente y la inclusión desde etapas tempranas.

- Vivencias de los valores en el aula.

- Valores que contienen los cuentos utilizados y que la población infantil practicaba en el aula y fuera de ella.

- La expresión de sus actitudes y aptitudes. 
- La independencia de la población infantil la cual aprende a desenvolverse con la guía de una persona adulta (facilitadora), desvinculándose de sus padres y madres.

Para otros la integración se produce en el "Trabajo en equipo, mediante actividades como agrupar por gustos de confites o armar rompecabezas. Por lo general hago más agrupación por confites. Y que los niños y niñas seleccionen sus propios responsables de equipo.”

3. El valor de los talleres dentro de la disciplina de la bibliotecología La bibliotecología es una profesión interdisciplinaria que requiere del apoyo de otras disciplinas, de los grupos sociales y de las comunidades para su crecimiento, por medio de los talleres se produce un contacto muy cercano entre estos sectores.

El objetivo de estos talleres es realizar un adecuado proceso de socialización en la población infantil y procurar el cierre de la brecha tecnológica e informacional, para garantizar un mejor futuro a las nuevas generaciones. Además, los talleres se utilizan como un recurso para atraer personas usuarias, en una época en la cual el usuario presencial ha disminuido en forma considerable, como dice una de las personas instructoras: "Los talleres se usan como promotores de la biblioteca y en general concretizan los objetivos de la misma, por medio de actividades didácticas que incluyan los recursos literarios y otros, que la biblioteca tiene a disposición de la sociedad." En este aspecto es importante destacar la función de la biblioteca para el apoyo académico. Su buen uso desde temprana edad contribuye a formar individuos más críticos y con una mentalidad investigativa.

Respecto al valor de los talleres, una persona instructora señala:

Los talleres son una fuente y un enlace de los usuarios con la biblioteca, son súper importantes para establecer comunicación y acercar desde los niños hasta los padres. Sí me parece importante que los talleres sean bien planificados, por estudiantes avanzados o profesionales y que se tome el tiempo necesario para la

\section{bibliotecas}


planificación y el desarrollo, que no se conviertan solo como un requisito con el que debe de cumplir la biblioteca, sino en un arma que se aproveche al máximo para diseminar información y formar lectores, hasta futuros investigadores.

Los talleres permiten mayor contacto con la comunidad y fortalecen el vínculo: biblioteca (universidad) -comunidad-sociedad. Como indica una de las personas instructoras: "Los mismos en mi persona generan mucha satisfacción, ya que al final de los mismos se visualizaron los frutos en los niños y las niñas y en los padres y madres de familia, ya que como mencioné anteriormente se brindó talleres a ambas poblaciones." Para una de las instructoras el trabajo interdisciplinario de la biblioteca infantil representó un laboratorio que le permitió poner en práctica los conocimientos aprendidos en su especialidad, atendiendo una población infantil de edad temprana.

\section{Discusión}

Esta investigación sobre el trabajo interdisciplinario en la Biblioteca Infantil Miriam Álvarez Brenes de la Escuela de Bibliotecología, Documentación e información de la Universidad Nacional, ha permitido visibilizar la labor realizada desde una biblioteca para contribuir a mejorar las condiciones sociales de una comunidad marginal.

El Manifiesto de la UNESCO (2002), entre otros aspectos, promulga que la biblioteca infantil ha de crear y consolidar los hábitos de lectura en la población infantil desde los primeros años, brindar posibilidades para el desarrollo personal, estimular la imaginación y la creatividad de esta población y de la población joven. Los espacios, servicios, actividades y colecciones de una biblioteca infantil están llamados a responder a las necesidades e intereses de la población infantil en sus diversas etapas evolutivas, para lograr en ella un desarrollo psicológico, intelectual, cultural y social.

Para la licenciada Sandra Alpízar (1992), pionera de la biblioteca infantil, la función de la biblioteca en la formación integral de los infantes, facilita: 
La adquisición del HÁBITO por la LECTURA, el aumento de la creatividad, la imaginación, la criticidad, el desarrollo de habilidades y destrezas psicomotoras, la capacidad de expresión, la concentración, la memoria, la socialización del niño y la capacidad para hacer uso del tiempo libre mediante actividades recreativas. De ahí, la importancia que deriva la tarea integradora y formadora que persigue la biblioteca infantil, en relación con la lectura, la educación formal, la búsqueda de información y la recreación para desarrollar los aspectos cognoscitivos, sociales y afectivos de los(as) niños(as) que la utilizan. (p.1)

Para cumplir adecuadamente con estas expectativas, en la Biblioteca Infantil Miriam Álvarez Brenes se ha integrado en el quehacer bibliotecológico, las teorías y los métodos de trabajo de otras disciplinas. En el transcurso de estos casi quince años de existencia de la biblioteca, la interacción entre la bibliotecología y otras disciplinas ha facilitado a la comunidad infantil de Jardines Universitarios 1 y 2 de Heredia, el acceso a la información que contribuye con el desarrollo de habilidades $\mathrm{y}$ competencias personales e intelectuales.

La biblioteca se ha caracterizado por atender las consultas del material bibliográfico de la colección infantil y, además, ha ofrecido talleres sobre diferentes temáticas a la numerosa población infantil que la ha visitado y disfrutado de un "viaje de ensueño en su vagón mágico. También ha integrado a sus actividades a las familias e imparte charlas a los padres $\mathrm{y}$ a las madres sobre temas que benefician su crecimiento personal y familiar. Este proyecto de biblioteca infantil se desarrolla a partir de una moderna visión del quehacer bibliotecológico: combina la función tradicional de proporcionar información con una función educativa de la comunidad, en un proceso de formación personal y de fomento de una cultura por medio de talleres y charlas (Rodríguez, 2003).

La caracterización de las actividades y de los actores de este proyecto de biblioteca ha permitido valorar la riqueza educativa que se produce en un ambiente donde interaccionan bibliotecólogos y especialistas de diversos campos, entre ellos, educadores, psicólogos y artistas. En el

\section{bibliotecas}


cuadro $\mathrm{N}^{\circ} 3$ se observa que en la biblioteca se ha producido la participación de especialistas desde diferentes áreas, que se han integrado a la labor formativa que realiza la bibliotecología, con los residentes de la comunidad de Jardines 1 y 2 de Heredia. En este cuadro se observa que del total de talleres el $34,9 \%$ fue impartido por personas instructoras con especialidad en bibliotecología y un $65,1 \%$ por personas instructoras de otras áreas: $23,3 \%$ de enseñanza preescolar, un 15,1\% enseñanza del inglés, 5,8\% de psicología, 5,8\% de enseñanza de la filosofía, 2,3\% de enseñanza del francés, $1,2 \%$ de artes plásticas.

La función educativa de la biblioteca infantil se ha forjado en el trabajo interdisciplinario de profesionales de diferentes áreas. Las habilidades, destrezas y conocimientos que se pretende desarrollar en los infantes y escolares que consultan la biblioteca infantil, se fortalecen con los talleres impartidos por personas instructoras de diferentes especialidades, quienes desde su área de estudio y trabajo interpretan las necesidades y las capacidades de la población infantil en forma integral.

Respecto a las personas instructoras de los talleres y las charlas impartidos en la Biblioteca Infantil Miriam Álvarez Brenes, se destaca el valioso aporte de estudiantes asistentes y de horas colaboración, que junto con estudiantes que han realizado su práctica supervisada, su trabajo comunal y hasta trabajado ad honorem, han fortalecido el escaso personal de la biblioteca. La mayor participación de los estudiantes en los talleres se ha producido desde su condición de estudiantes asistentes y de horas colaboración, los responsables del taller son los primeros, los segundos son colaboradores de la actividad.

En su mayoría, los talleres han sido impartidos por el personal de planta de la biblioteca y por estudiantes, contratados por horas asistente, de las especialidades de educación o enseñanza, bibliotecología y psicología. Los estudiantes que hacen horas colaboración no acostumbran impartir talleres, su participación es de apoyo a la persona instructora. Se encuentran también estudiantes que han laborado en modalidades de curso optativo, trabajo comunal, ad honorem, práctica supervisada, pero que han tenido muy poca participación en la realización de los talleres. 
Es precisamente esta participación de los estudiantes de diversas disciplinas lo que ha impregnado a los talleres y a las charlas un carácter de interdisciplinariedad. Como se estableció en el Marco Teórico la interdisciplinariedad se presenta cuando se producen interacciones que relacionan diversas disciplinas entre sí y estas interacciones entre el personal de bibliotecología con el de otras disciplinas, como la educación y la psicología, solo se producen en la realización de los talleres y las charlas, únicas actividad de la biblioteca que se llevan a cabo en forma conjunta.

Llama la atención que no se encuentre documentada la participación conjunta en la realización de los talleres de estudiantes de dos o más especialidades. En la mayoría de los casos los talleres se imparten por una sola persona instructora, cuando ha habido participación de más de una persona, estas han sido de la misma especialidad. Solo con los estudiantes de apoyo a los talleres se presenta la participación de más de una disciplina en la instrucción de los talleres y se ha dado entre la disciplina de la bibliotecología y la psicología.

La falta de representación de varias disciplinas durante el proceso de instrucción de los talleres se puede considerar una limitación a la interdisciplinariedad, condición que si es evidente en la organización del taller, pero no en la instrucción conjunta de la temática.

Con respecto a los talleres y a las charlas impartidos en la biblioteca, en el período del año 2000 al 2009 se impartieron en total 90 talleres, 86 a la población infantil y 4 a las familias de la comunidad de Jardines 1 y 2 de Heredia. También se realizaron 10 charlas, 1 a la población infantil y 9 a las familias. Del total de talleres impartidos se destacan los relacionados con idiomas con un 18,6\%, promoción de la lectura un $16,3 \%$, motivos navideños el $16,3 \%$ y apresto escolar el $12,8 \%$, estos temas comprenden el $64,4 \%$ de los talleres.

Los talleres han abarcado una gran amplitud de temáticas, desde la adquisición de destrezas motrices en la población infantil, conocimientos sobre el inglés, sobre los libros y la lectura, valores personales, hábitos de higiene hasta los adornos navideños y los temas ecológicos. Es importante destacar que estos talleres están orientados a esta po-

\section{bibliotecas}


blación, pero también se han impartido a los padres y a las madres de ella, talleres sobre el fortalecimiento de la familia y la participación de los progenitores en el desarrollo integral de los infantes y, otros temas, como el estímulo de conductas no violentas.

Las actividades de formación de la biblioteca se han extendido también a las familias de la comunidad de Jardines 1 y 2 de Heredia, en el período del estudio se realizaron cuatro talleres y 11 charlas a esta población, con el propósito de que la formación de la población infantil sea apoyada también desde sus hogares.

Los talleres han cumplido un objetivo muy importante en la comunidad usuaria, contribuyendo para que en la población infantil se realice un adecuado proceso de socialización. Al mismo tiempo se promueve el cierre de la brecha tecnológica e informacional, con talleres sobre las nuevas tecnologías y diferentes idiomas, principalmente, el inglés, para garantizar un mejor futuro a las nuevas generaciones.

\section{Conclusiones}

1. La Biblioteca Infantil Miriam Álvarez Brenes se origina en la preocupación por los que menos tienen, es la respuesta de una sociedad interesada en crear instituciones inclusivas e intenta desde las herramientas que proporciona la bibliotecología, contribuir con la formación integral de la población de las clases menos favorecidas de las comunidades aledañas a la Universidad Nacional.

2. La biblioteca ha realizado durante todos estos años una labor social de gran importancia en la provincia de Heredia: la población infantil ha iniciado su proceso de socialización en un espacio de libros y juegos, aprendiendo desde muy temprana edad el valor de la lectura y de los juegos tradicionales; además, han adquirido conocimientos y destrezas para su desarrollo personal y social. Sus padres y madres también se han formado y compartido esta maravillosa experiencia con sus hijos.

3. La biblioteca, por su parte, ha innovado y evolucionado a una forma diferente de la práctica bibliotecológica, no es solo un lugar 
donde se prestan libros, sino donde se juega y se imparten talleres y charlas para adquirir una mejor formación, en espera de que toda la población infantil sea un mejor ciudadano en el futuro. En este aspecto es importante destacar la función de la biblioteca para el apoyo académico, su uso desde temprana edad, contribuye a formar individuos más críticos y con una mentalidad investigativa.

4. En la biblioteca se ha producido una gran variedad de aportes de trabajo desde diferentes áreas de especialidad, que se han integrado a la labor formativa que realiza la bibliotecología. La función educativa de la biblioteca infantil por medio de los talleres, ha sido reforzada por el trabajo de los estudiantes de diferentes disciplinas académicas de la Universidad Nacional: psicología, educación, bibliotecología, idiomas, entre otras. Las habilidades, destrezas y conocimientos que se pretenden desarrollar en los infantes y escolares que consultan la biblioteca infantil, se fortalece con los talleres impartidos por grupos de profesionales de diferentes especialidades, quienes desde su área de estudio y trabajo interpretan las necesidades y las capacidades de la población infantil en forma integral

5. La disciplina de la bibliotecología se muestra fortalecida, tanto por la labor formativa que se ha desarrollado en la comunidad, como por la integración que se ha producido con otras especialidades, donde los recursos y saberes propios de la disciplina bibliotecológica se han complementado con los saberes de otras disciplinas afines, como la psicología y la educación, y de esta forma se ha cumplido una mejor función social en beneficio de la comunidad.

6. En los talleres que se imparten en la biblioteca se destacan varios factores: la formación académica y nivel educativo de las personas instructoras, la biblioteca se convierte en un espacio dinamizador de aprendizajes con la participación activa del estudiantado que se vincula como estudiante asistente o estudiante investigador y la presencia del trabajo en equipo con la presencia de factores de organización, planificación, recursos materiales y físicos, difusión y sistematización de los resultados obtenidos. Se puede decir que todos los

\section{bibliotecas}


actores involucrados en el proceso se ven beneficiados: la población infantil, padres de familia y estudiantes de la universidad.

7. En cuanto a las fuentes documentales fue un trabajo de investigación que requirió mucho análisis de estas, por el periodo comprendido en el estudio (10 años). El tiempo fue corto especialmente también para el contacto y localización de las personas instructoras.

\section{Recomendaciones}

1. La labor formativa de la Biblioteca Infantil Miriam Álvarez Brenes debe mantenerse y fortalecerse. Es necesario que se le proporcione el apoyo financiero requerido para la adquisición de materiales y equipo tecnológico moderno para que pueda satisfacer adecuadamente las demandas de una sociedad que ha avanzado muy rápidamente. Es necesario dotarla del personal suficiente para que los talleres y las demás actividades se realicen de la mejor forma y también se puedan incrementar todas estas actividades formativas para la población infantil y para sus familias. Las instructoras entrevistadas expresan ciertas carencias de materiales que limitan el desarrollo de los talleres que debieran de proporcionarse para mejorar el servicio de la biblioteca.

2. Los talleres permiten mayor contacto con la comunidad y fortalecen el vínculo: biblioteca (universidad) -comunidad-sociedad. Para una de las personas instructoras el trabajo interdisciplinario de la biblioteca infantil representó un laboratorio que le permitió poner en práctica los conocimientos aprendidos en su especialidad atendiendo a una población infantil. Por esta razón se debiera de promocionar la investigación de las diferentes carreras de la Universidad en la biblioteca y en la población que la visita.

3. La perspectiva interdisciplinaria en las actividades de la biblioteca se puede extender a los trabajos de graduación de los estudiantes, si la escuela define temas de investigación para que estudiantes de bibliotecología los realicen en forma conjunta con estudiantes de otras disciplinas como informática, educación, psicología, etc. 


\section{Referencias}

Alpízar, S. (1992). Sistema de bibliotecas infantiles, su proyección en la educación costarricense. El Manejo de la información en Áreas Especializadas en Costa Rica. Revista Bibliotecas, X (2).

Alpízar, S. (2001). Proyecto de extensión Biblioteca Infantil "Miriam Álvarez Brenes". Heredia, C.R.: UNA.

Alpízar, S. (1997). Un vagón de tren y dos contenedores han albergado durante un año la primera Biblioteca Infantil en Heredia. Boletín Bibliotecas, XV (2).

Avendaño, C. (2008). Creación de una bebeteca: manual de mejores prácticas. Universidad de Puerto Rico, Recinto de Río Piedras Escuela Graduada de Ciencias y Tecnologías de la Información. Obtenido en http://sites.google.com/site/manualdebebeteca/

Barboza, L., Segura, G. y Vargas, F. (2009). Percepción y opinión de los niños y niñas, padres y madres de familia en relación con los servicios y productos de información que ofrece la Biblioteca Infantil "Miriam Álvarez Brenes" de la Universidad Nacional. Revista Bibliotecas, XVII (2).

Barboza, L. (2008). Estudio de usuarios(as) de las comunidades Jardines 1 y Jardines 2 que atiende la Biblioteca Infantil "Miriam Álvarez Brenes". Heredia, C.R.: UNA-EBDI.

Diefenbach, T. (2008). "Are case studies more than sophisticated storytelling? Methodological problems of qualitative empirical research mainly based on semi-structured interviews". Quality \& Quantity, 43.

Escuela de Bibliotecología Documentación e Información (EBDI). Proyecto de extensión de la Biblioteca Miriam Álvarez. Heredia, C.R.: UNA. Obtenido en http://www.una.ac.cr/Catalogo/Catalogo/FFL/7BIBLIOTECA.pdf

Escuela de Bibliotecología Documentación e Información (EBDI). Informe de labores 1996-1997 (primeros seis meses) de la Biblioteca Miriam Álvarez. Heredia, C.R.: UNA.

\section{bibliotecas}


Escuela de Bibliotecología Documentación e Información (EBDI). Informes de labores 1997-2009 de la Biblioteca Miriam Álvarez. Heredia, C.R.: UNA.

García, R. (2006). Sistemas Complejos. Conceptos, métodos y fundamentación epistemológica de la investigación interdisciplinaria. Barcelona: Gedisa.

Gorbea, S. (2006). Perspectivas interdisciplinarias de los Estudios métricos de la información. Problemas y métodos de investigación en bibliotecología e información. Una perspectiva interdisciplinaria. México: UNAM.

Hasan, H. (2009). Questionnaries and Interviews in Educational Researches. Journal of Graduate School of Social Sciences, 1.

Jiménez, Y. (2009a). Aporte de la Biblioteca Infantil "Miriam Álvarez Brenes" y de la familia en la formación de los hábitos de lectura de la población infantil de Jardines Universitarios I y II, Heredia, Costa Rica: año 2007. Tesis de graduación para optar el grado de Licenciatura en Bibliotecología y Documentación. Heredia, C.R.: UNA.

Jiménez, Y. (2009b). Aporte de la biblioteca infantil Miriam Álvarez Brenes y de la familia en la formación de los hábitos de lectura de la población infantil de jardines universitarios I y II, Heredia, Costa Rica: año 2007. Revista Bibliotecas, XVII (2).

Miranda, A. (2003). Presentación de candidatura: Premio a la innovación académica. Proyecto Biblioteca Infantil "Miriam Álvarez Brenes". Folleto no publicado. Heredia, C.R.: UNA-EBDI.

Rodríguez, A. (2006). Las ciencias, la bibliotecología y la interdisciplina. Problemas y métodos de investigación en bibliotecología e información. Una perspectiva interdisciplinaria. UNAM: México.

Rodríguez, K. (2003a). Diagnóstico de las comunidades Jardines Universitarios 1 y 2 . Heredia, C.R.: UNA-EBDI. No publicado.

Rodríguez, K. (2003b). Bases para la creación de una biblioteca infan- 
til acorde a los retos de la sociedad actual: la experiencia de la Biblioteca Infantil Miriam Álvarez Brenes. Trabajo presentado en el Congreso Internacional "Metáforas de la Sociedad de la Información en el contexto Latinoamericano y del Caribe”, 1618 junio. Heredia, C.R.: UNA-EBDI.

Ten Have, P. (2004). Understanding Qualitative Research and Ethnomethodology. Londres: SAGE Publications.

UNESCO-IFLA (1994). Manifiesto de la UNESCO sobre la biblioteca pública. Obtenido en http://unesdoc.unesco.org/images/0011/001121/112122so.pdf

Vargas, F. (2000). La biblioteca un universo por descubrir. Ponencia. Seminario-taller "Libros alas a la creatividad". San José, C.R.: CENAT.

Vargas, F. (2006). Los padres y madres de familia usuarios que atiende la Biblioteca Infantil, Jardines Universitarios 1 y 2. Documento borrador. Heredia, C.R.: UNA-EBDI.

Zárate, A. (2008). Diseño de una propuesta didáctica para la promoción de la lectura que favorece el área emocional en los niños y niñas de 7 a 9 años que asisten a la biblioteca infantil Miriam Álvarez Brenes de la Universidad Nacional. Tesis para optar el grado de licenciatura en Pedagogía con énfasis en I y II ciclo de la Educación General Básica. Heredia, C.R.: UNA-CIDE.

\section{Notas de la autora}

Máster María Marjorie Mora Valverde: Investigadora y Académica de la Escuela de Bibliotecología, Documentación e Información (EBDI), Universidad Nacional (Costa Rica). Correo electrónico: marjorie.mora.valverde@una.cr

1 Un agradecimiento muy especial a todas las personas que colaboraron en la presente investigación; a la máster Flor Vargas por compartir todos los conocimientos que ha acumulado durante sus casi catorce años como encargada de la biblioteca; a todas las personas instructoras que muy amablemente brindaron la información solicitada para que esta investigación se pudiera realizar; a la estudiante Valezka Rodríguez por su valioso apoyo en la recopilación de los datos sobre los talleres realizados y los usuarios atendidos en la biblioteca.

El informe completo puede consultarse en la página electrónica de la EBDI en la dirección http://www. una.ac.cr/bibliotecologia/index.php?option=com_content\&task=view\&id=250\&Itemid=504)

\section{bibliotecas}

